\title{
Calidad Integral del Agua Superficial en la Cuenca Hidrológica del Río Amajac
}

\author{
Jesús P.A. Álvarez ${ }^{1,2}$, Juan E.R. Panta ${ }^{1}$, Carlos R. Ayala ${ }^{1}$ y Elizabeth H. Acosta ${ }^{3}$ \\ (1) Colegio de Postgraduados Campus Montecillo, Km 36.5 Carretera México-Texcoco, Estado de \\ México, C.P. 56230 (e-mail:jesusaa@colpos.mx, jerpkike@colpos.mx, cara@colpos.mx ) \\ (2) Instituto Nacional de Investigaciones Forestales Agrícolas y Pecuarias-Campo Experimental \\ Delicias, Km. 2, Carretera Delicias-Rosales, Cd. Delicias, Chihuahua-México. C.P. 33000 \\ (e-mail: amado.jesus@inifap.gob.mx) \\ (3) Universidad Autónoma de Chapingo, Depto. de Suelos, Área de Recursos Naturales, Chapingo, \\ carretera México- Texcoco Km. 38.5, Texcoco-Estado de México (e-mail: elizahac@yahoo.com.mx)
}

\begin{abstract}
Resumen
Se presenta un estudio integral sobre la calidad del agua superficial en los diferentes almacenamientos y corrientes de la cuenca hidrológica del río Amajac. Se identifican los problemas asociados con contaminantes específicos y se establecen alternativas de solución que sirvan como base para programas y políticas de ordenamiento de los recursos hídricos. Se seleccionaron cuatro presas, una laguna y cinco ríos, donde se midió el caudal, la velocidad del agua y el tirante máximo. También se determinaron las principales características físicas, químicas y microbiológicas de las aguas: oxígeno disuelto, coliformes fecales, nitrógeno, fósforo, sulfatos, carbonatos bicarbonatos, cloro y manganeso. De acuerdo con los resultados, se concluye que el agua del río en Tulancingo está fuertemente contaminada.
\end{abstract}

Palabras clave: calidad de agua, contaminación de ríos, cuenca hidrológica, agua superficial

\section{Integral Quality of Surface Water in Rio Amajac Watershed}

\begin{abstract}
This paper presents an integral study on the quality of the superficial waters in ponds and streams of the watershed of Amajac river. The problems associated to specific pollutants are identified and alternative solutions are detailed to be used in public policies of water resources. Four ponds, one lake and five streams were considered in the study and runoff volume, water speed, and maximum depth were measured. Also, the main physical, chemical and biological parameters were determined: dissolved oxygen, fecal coli forms, nitrogen, phosphorus, sulphate, carbonate, bicarbonate, chloride, and manganese. According to the results, it is concluded that water of the river in Tulancingo city is strongly contaminated.
\end{abstract}

Keywords: water quality, river pollution, watershed, surface water 


\section{INTRODUCCIÓN}

La calidad del agua está afectada por diversos factores como los usos del suelo, la producción industrial y agrícola, el tratamiento que se le da antes de ser vertida nuevamente a los cuerpos de agua, y a la cantidad misma del agua de los ríos y lagos, ya que de ésta depende su capacidad de purificación. Calidad del agua, es el termino ampliamente usado, sin embargo la cuantificación científica resulta bastante importante y esta solución es una estrategia básica en el desarrollo de los fundamentos para el manejo de los recursos hídricos (Hakanson et al., 2000).

La percepción que tiene la comunidad sobre la salud pública, se basa en las normas establecidas para la reutilizar el agua residual (Taylor et al., 2006), ellos indican que se debe tener poco contacto humano con los tratamientos aplicados a los efluentes, sin embargo el rehúso del recurso con fines agrícolas ha sido aceptado en el 99\%, de los casos en el Sur de Australia. Jones (2006), indica que las estrategias de limpieza de aguas residuales fueron: a)promover la calidad del agua al límite inferior particularmente la DBO, y el amonio; b) promover o aumentar los tratamientos primarios de las aguas residuales; c)imponer un estricto control en las descargas industriales.

Jones et al. (2002), utilizaron un modelo numérico simple para evaluar las opciones potenciales de ingeniería. Ellos registraron mejoras significativas en la calidad del agua no marina del río, al aplicar tratamientos secundarios en lo alto de las descargas del estuario y tratamiento primario para la parte baja del estuario en Birkenhead/Liverpool, y se pudo salvar los problemas de oxígeno disuelto. Sharma y Ashwath (2006), consignaron plantas de tratamientos para efluentes de irrigación en Australia tales como Warribee, Wagga, Shepparton, y otros sitios donde se ha mejorado su calidad de agua sobresaliendo la práctica de los defensores de la degradación ecológica por el contenido de los de los contaminantes en el suelo, además de crear oportunidades para la producción comercial de biomasa.

La cuenca hidrológica del Río Amajac se localiza en el Estado de Hidalgo, México; abarca una superficie de $6,904 \mathrm{~km}^{2}$. Las entradas anuales de agua dentro del área de la región aportadas por la precipitación $(972 \mathrm{~mm})$ son de alrededor de 6,710.2 millones de $\mathrm{m}^{3}$. La longitud total del cauce principal es de $310 \mathrm{~km}$ (Panta et al., 2006). La población total para el año 2005, era de 505,263 habitantes distribuidos en 1,388 localidades, de las cuales 1,313 pertenecen al estado de Hidalgo, 51 a San Luís Potosí, 18 a Veracruz, y 6 al estado de Puebla; 15 son consideradas como localidades urbanas ( de 2500 a 20,000 habitantes) y 1,373 como localidades rurales (< de 2500 habitantes) (INEGI, 2005).

En el área se practica agricultura con agua de lluvia y de riego, sobresaliendo los cultivos de maíz, fríjol, trigo y avena forrajera, otros de menor importancia, son el café y la naranja, los cuales están en función de las condiciones de clima. Estas áreas agrícolas están expuestas al proceso de deterioro por el uso continuo del suelo. La ganadería se practica en toda la cuenca destacando el pastoreo de bovinos, ovinos y caprinos, lo que conlleva a un deterioro de los estratos herbáceos y arbóreos propiciando la erosión del suelo, la pérdida de especies vegetales y perturbación del hábitat de las especies animales silvestres. La actividad forestal maderable presenta un alto grado de concentración en los municipios de Acaxochitlán. Agua Blanca de Iturbide y Zacualtipán de los Ángeles, los cuales se localizan al sureste y al este del área de estudio (Ayala et al., 2006).

Las combinaciones del rápido crecimiento de la población complementado con las actividades urbanas, agrícolas, pecuarias, forestales, además de las condiciones físicas y químicas del terreno en general ha resultado en un fuerte deterioro de la calidad del agua dentro de la cuenca. La mayor fuente de contaminación se produce por aguas residuales, vertidas al río Tulancingo en la ciudad con el mismo nombre las cuales no tienen depuradora de ningún tipo.

Los parámetros reportados por Acosta et al. (2006), indican que los problemas de contaminación fueron de 368600 ufc $\mathrm{mL}^{-1}$, de bacterias mezo filas aeróbicas en la presa la Esperanza, hasta 7 , 933,333 ufc $\mathrm{mL}^{-1}$ de dichas bacterias en la salida de la ciudad de Tulancingo; las bacterias coliformes totales fluctuaron de 1,618 a 1, 753 NMP $100 \mathrm{~mL}^{-1}$, para los mismos puntos y de 1,220 a 2,400 NMP $100 \mathrm{~mL}^{-1}$ de bacterias coliformes fecales, para los mismos sitios. El oxígeno disuelto en ésta área fue 
de $0 \mathrm{mg} \mathrm{L}^{-1}$. Los sólidos totales disueltos fluctuaron de $927 \mathrm{mg} \mathrm{L}^{-1}$ a 12,970 $\mathrm{mg} \mathrm{L}^{-1}$ en la misma zona, mientras que los sólidos disueltos se cuantificaron de 65 a $86 \mathrm{mg} \mathrm{L}^{-1}$, datos que sobrepasan los valores máximos establecidos por la Norma Oficial Mexicana NOM-001-ECOL-1996 (Secretaría de Economía 1996).

El objetivo del presente trabajo desarrollar un estudio integral de la calidad del agua, que incluya variables químicas, físicas y microbiológicas en los diferentes almacenamientos y corrientes, a fin de identificar problemas asociados con contaminantes específicos, establecer alternativas de solución de acuerdo a la problemática regional, que sirvan de base para programas y políticas de ordenamiento de los recursos hídricos.

\section{MATERIALES Y METODOS}

\section{Zona de estudio}

La zona de estudio esta ubicada en los Distritos de Desarrollo Rural de Zacualtipan, Pachuca y Tulancingo del Estado de Hidalgo, pertenece a la cuenca Alta del Río Panuco y esta incluida en la Región Hidrológica Prioritaria de la CONABIO, denominada Confluencias de las Huastecas en el Estado de Hidalgo (Fig. 1). Existe una gran diversidad biológica vegetal y animal (INEGI, 2003) también se practica la actividad industrial, minera, ganadera silvícola y turística. El clima es semicálido húmedo con abundantes lluvias en verano, la precipitación pluvial fluctúa entre 700 y $3000 \mathrm{~mm}$, la temperatura media anual de $12-26^{\circ} \mathrm{C}$.

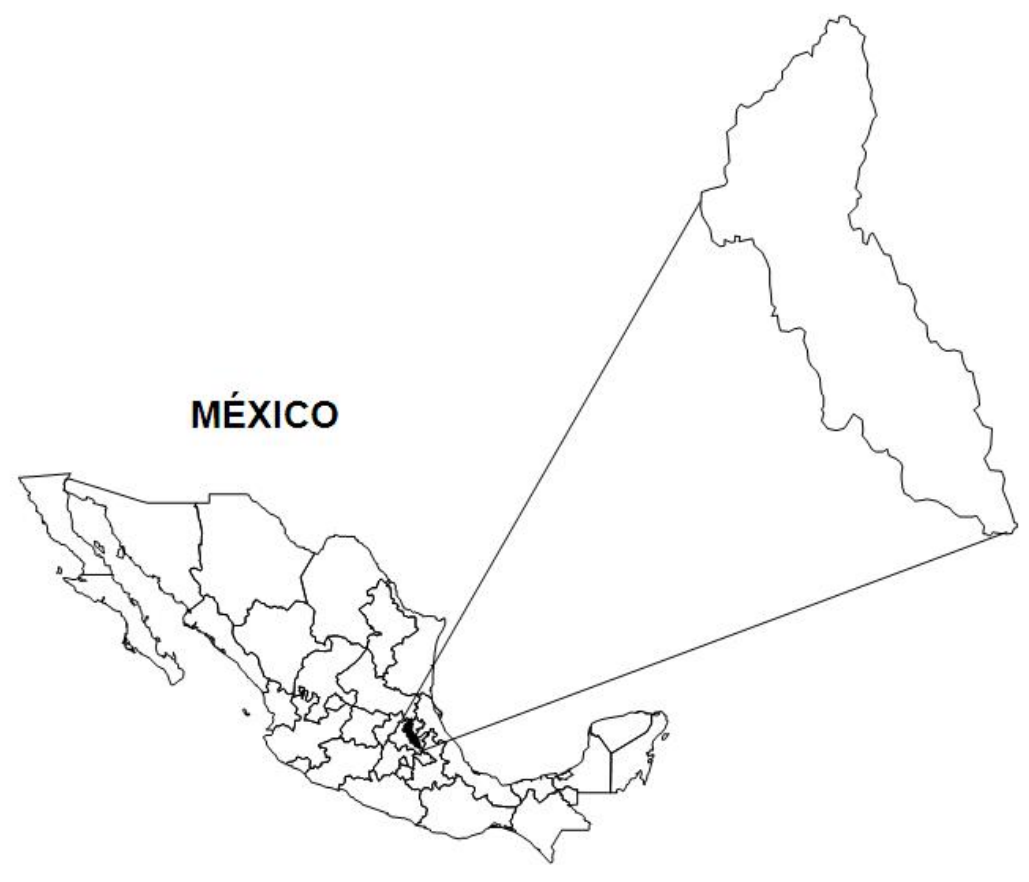

Fig. 1: Ubicación del área de estudio

De acuerdo a las condiciones naturales de la cuenca, se seleccionaron 17 sitios de muestreo: considerando 1) la estación de aforos del río Venados, 2) Laguna de Metztilán, 3) río Almolón, 4) río Amajac (San Juan Amajac), 5) río Amajac (SJA-2), 6) río Amajac (SJA-2), 7) río Amajac (Boca de León), 8) río Amajac, antes de Tamazuchale, 9) río Moctezuma en Tamanzunchale, 10) río Amajac (río Amajac + Moctezuma), 11) Presa El Cedral, (Pachuca, Hgo.), 12) Presa La Estancuela (Pachuca Hgo.), 13) Presa El Comalillo (inicios del río Amajac), 14) río Tulancingo, 15) Presa La Esperanza (cd. De Tulancingo), 16) río Tulancingo (centro de la ciudad), 17) río Tulancingo (Distrito de Desarrollo Rural); su localización exacta se muestra en la Fig. 2. Mediante el empleo de un geoposicionador portátil (GPS), se registró la altitud, latitud y longitud de cada punto dentro de la cuenca hidrológica. 


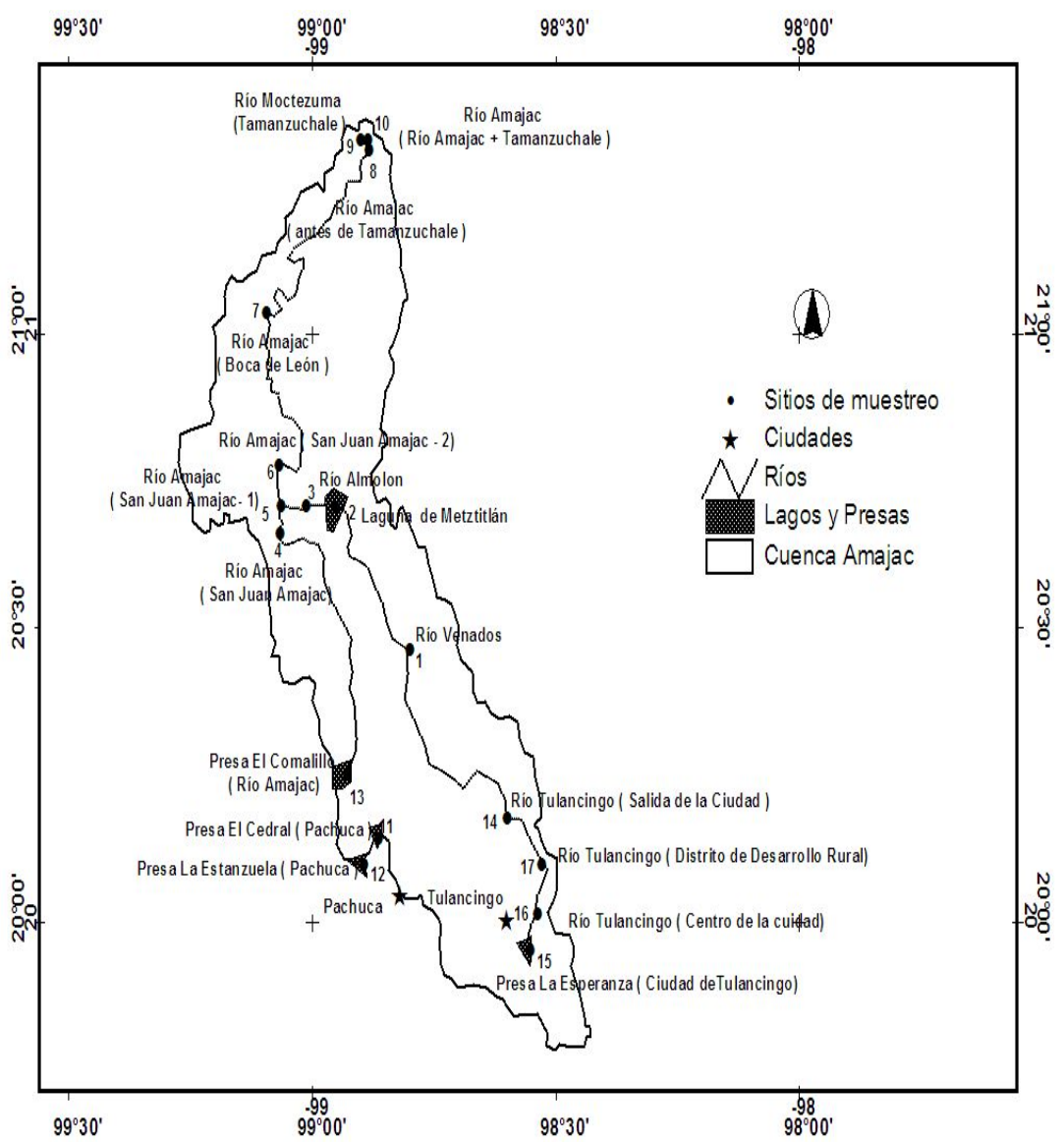

Fig. 2: Localización de los sitios de muestreo dentro de la cuenca del Río Amajac,

Parámetros para determinar la cantidad y calidad integral del agua

La cantidad del agua (parámetros hidráulicos) en los tributarios y ríos de la cuenca Amajac, se determinó utilizando la metodología descrita por Briones y García (1997). Se tomaron tres muestreos de agua (Primero Septiembre 2005, segundo noviembre del 2005, inmediatamente después que paso el Huracán Vilma y el tercero, en Enero del 2006).

De las muestras tomadas se obtuvieron muestras para cada tipo de análisis, y se prepararon según las normas respectivas. Se analizó la conductividad eléctrica, sólidos totales disueltos, temperatura y $\mathrm{pH}$, in situ con un potenciometro multifuncional (Hanna Instruments $\mathrm{pH} / \mathrm{CE} / \mathrm{TSD} /{ }^{\circ} \mathrm{C}$ meter), La demanda bioquímica de Oxígeno( NMX-AA-028), N total (NMX-AA-026), P total (NMX-AA-029), elementos traza: $\mathrm{Pb}, \mathrm{Zn}, \mathrm{Ni}, \mathrm{Cu}, \mathrm{Cd}$, (NMX-AA-51) y el número más probable de bacterias coniformes totales (NMP $100 \mathrm{ml}^{-1}$ ), con caldo laurel sulfato y coniformes fecales (caldo Brilla), en el laboratorio , siguiendo los lineamientos establecidos en el anexo 1 de la NOM-001-ECOL-1996 Secretaría de Economía (1996). Se utilizó la técnica estadística del análisis de Componentes Principales, (correlación lineal de Pearson), con el objeto de observar la influencia de los parámetros estudiados sobre la calidad integral del agua usando los procedimientos del paquete estadístico SAS (SAS, 2001).

\section{RESULTADOS Y DISCUSION}

\section{Parámetros hidráulicos}

La cantidad de agua promedio durante el período del estudio, consideró un caudal que fluctuó entre $0,020 \mathrm{~m}^{3} \mathrm{seg}^{-1}$, en los inicios del río Tulancingo, inmediatamente después de la presa La Esperanza, hasta $87,056 \mathrm{~m}^{3} \mathrm{seg}^{-1}$, donde se juntan las aguas del río Amajac, con las del río Moctezuma el cual 
lleva aguas desde la Ciudad México (Tabla 1). En el recorrido total del cauce principal, se cuantificaron 66 corrientes de primer orden, 18 tributarios de segundo orden, cuatro tramos de tercer orden, lo que equivale a que la corriente principal desde los inicios del río Tulancingo, hasta la cuidad de Tamanzunchale en San Luís Potosí se establezca de cuarto orden. La velocidad del agua fluctuó desde $0,0 \mathrm{~m} \mathrm{seg}^{-1}$, en la presa El Cedral, ya que no tenia volúmenes excedentes, (en las presas y lagos se refiere al volumen captado, al caudal y velocidad del agua de salida), hasta 1,3 $\mathrm{m} \mathrm{seg}^{-1}$, en el río Amajac después de que sus aguas se juntan con las del río Moctezuma al cruzar por la ciudad de Tamanzuchale, San Luís Potosí, lo que se consideró como el final de la cuenca Amajac. Otra de las características hidráulicas medidas dentro del cauce principal, fue el tirante, o carga de agua, Sobresaliendo por su valor $(6,5 \mathrm{~m})$, la profundidad de la laguna de Metztitlán, la cual por sus condiciones físicas naturales sirve como regulador de inundaciones a los poblados que se localizan aguas abajo de la corriente principal, cuyo nombre en ese tramo se convierte en río Almolón.

László et al. (2007), reportan que los directivos sobre el manejo del agua en Estados Unidos, trabajan para alcanzar un buen estado de las aguas superficiales, antes del año 2015, por lo que se deberá establecer un sistema que incluya una buena vigilancia, operación e investigación, con elementos hidráulicos, morfológicos, biológicos y químicos. En Hungría, la red de supervisión estándar para la calidad del agua superficial se ha fusionado para los ríos y lagos grandes, pero faltaba hacerlo para los cuerpos pequeños y los arroyos como los encontrados en las corrientes del Nagy en las montañas de Mátra, los cuales se muestrearon durante tres años (Mayo del 2002 a Mayo del 2005).

Tabla 1: Parámetros hidráulicos en los sitios de muestreo dentro de las subcuencas de los ríos Amajac, Tulancingo, Venados, Almolón y Moctezuma.

\begin{tabular}{lccc}
\hline \multicolumn{1}{c}{ Sitios de Muestreo } & Tirante $(\mathrm{m})$ & $\mathrm{V}\left(\mathrm{m} \mathrm{seg}^{-1}\right)$ & Caudal $\left(\mathrm{m}^{3} \mathrm{seg}^{-1}\right)$ \\
\hline 1.- Río Venados & 0,56 & 0,65 & 1,132 \\
2.- Laguna de Metztitlán & 6,50 & 0,80 & Volumen $=52,0 \mathrm{Mm}^{3}$ \\
3.- Río Almolón & 1,50 & 1,275 & 1,425 \\
4.- Río Amajac ( S J Amajac) & 0,40 & 0,84 & 2,731 \\
5.- Río Amajac ( S J Amajac- 1) & 0,39 & 0,86 & 3,050 \\
6.- Río Amajac ( S J Amajac - 2) & 0,39 & 0,86 & 3,050 \\
7.- Río Amajac ( Boca de León ) & 1,00 & 0,71 & 12,906 \\
8.- Río Amajac ( antes de Tamanzunchale ) & 1,30 & 0,88 & 16,166 \\
9.- Río Moctezuma (Tamanzuchale ) & 2,20 & 1,02 & 70,890 \\
10.-Río Amajac ( Amajac + Moctezuma) & 3,50 & 1,32 & 87,056 \\
11.-Presa El Cedral ( Pachuca ) & 4,80 & 0,0 & Volumen $=0,56 \mathrm{Mm}^{3}$ \\
12.-Presa La Estanzuela ( Pachuca ) & 1,74 & 0,15 & Volumen $=0,45 \mathrm{Mm}^{3}$ - \\
13.-Presa El Comalillo ( Río Amajac) & 56,2 & 0,25 & Volumen $=6,2 \mathrm{Mm}^{3}$ - \\
14.- Río Tulancingo ( Salida de Ciudad) & 0,05 & 0,30 & 0,027 \\
15.- Presa La Esperanza ( Ciudad Tulancingo) & 3,68 & 0,22 & Volumen $=3,92 \mathrm{Mm}^{3}$ - \\
16.- Río Tulancingo ( Centro ciudad) & 0,30 & 0,81 & 0,020 \\
17.- Río Tulancingo ( Distrito Desarrollo Rural) & 0,05 & 0,22 & 0,150 \\
\hline
\end{tabular}

\section{Temperatura}

Los resultados obtenidos sobre este parámetro indican una relación inversamente proporcional entre la temperatura y la altura sobre el nivel del mar del sitio de muestreo, o sea que a mayor altura menor temperatura del agua, teniendo así que en el área de Tulancingo el valor fue de $18,3^{\circ} \mathrm{C}$, hasta $21,6^{\circ}$ $\mathrm{C}$, dependiendo de la fecha de muestreo, en el centro de la cuenca, (donde se une el río Almolón con el Amajac), el rango de temperatura fluctuó desde 24,7 hasta $25,2^{\circ} \mathrm{C}$; mientras que a la salida de la cuenca, la temperatura promedio del agua en el río varió desde 26,2 hasta $26,9^{\circ} \mathrm{C}$. Esta información explica la presencia de material vegetal, desde la Presa la Esperanza, río Venados, 
Laguna de Meztitlán, río Almolón, Boca de León, hasta la ciudad de Tamanzunchale, en diferentes proporciones.

Al respecto en un estudio hecho por Justic et al. (1996), indicaron que la contaminación térmica incrementa la solubilidad de ciertos compuestos químicos, y en general disminuye la solubilidad de gases, especialmente la del oxígeno disuelto, reportando también que a mayor temperatura, mayor evaporación del agua. En otro trabajo Foreman et al. (1997), registraron que el agua caliente puede afectar la vida en el agua, lo cual resulta en un cambio de la composición fisiológica de las especies acuáticas; el calentamiento puede acelerar los procesos biológicos en plantas y animales por disminución del oxígeno en el agua. En otro reporte, Sawidis (1997), comenta que la vulnerabilidad para el incremento de enfermedades y cambios en la producción de algas e invasión de organismos destructivos es expectante; cada pequeño cambio de temperatura en un cuerpo de agua puede conducir a lo largo del tiempo al fin de una especie y a el origen de otra vida silvestre, que originalmente estuvo presente cerca de la descarga de la fuente y atraer otra especie en su lugar. Frutos (1998), en un estudio hecho en Argentina, registró que la abundancia del zooplancton fue correlacionado positivamente con los factores temperatura, transparencia y fitoplancton.

\section{Sólidos Inorgánicos}

Dentro de los sólidos inorgánicos se consideró a carbonatos, bicarbonatos, sulfatos, cloruros, fósforo total, nitrógeno total y los elementos potencialmente tóxicos (cobalto, níquel, fierro, cobre, zinc, manganeso, cadmio, y plomo). Todos los valores obtenidos en la cuenca del río Amajac, sobrepasan los valores límites establecidos en la Norma Oficial Mexicana, (100 mg L $\left.{ }^{-1}\right)$, NOM-001-SEMARNAT1996 Secretaría de Economía (1996). Lo cual indica contaminación del agua de diferentes formas. Es importante registrar que en el desglose de los componentes estudiados, como se muestran en la Tabla 2; los Bicarbonatos, constituyen el 92,38 \% del total de los sólidos inorgánicos; y que los valores más altos $\left(1122,37 \mathrm{mg} \mathrm{L}^{-1}\right)$ fueron consignados en la zona más contaminada, equivalente a el área adyacente de la ciudad de Tulancingo.

En un trabajo reportado por Kretschmr et al. (2002), establecieron los valores generales de calidad del agua para riego agrícolas dependiendo del grado de restricción, menos de $90 \mathrm{mg} \mathrm{L}^{-1}$ de bicarbonatos, se pueden utilizar sin ningún impedimento, de 90 a $500 \mathrm{mg} \mathrm{L}^{-1}$ la limitación es moderada, mientras que en un rango mayor de $500 \mathrm{mg} \mathrm{L}^{-1}$ el permiso es severo.

Tabla 2: Valores promedio de sólidos inorgánicos (S I) $\mathrm{mg} \mathrm{L}^{-1}$ en aguas de la cuenca hidrológica del Amajac

\begin{tabular}{cccccccccccccc}
\hline LOC & $\mathrm{Ni}$ & $\mathrm{Cu}$ & $\mathrm{Zn}$ & $\mathrm{Mn}$ & $\mathrm{Pb}$ & $\mathrm{Cd}$ & $\mathrm{Nt}$ & $\mathrm{Pt}$ & $\mathrm{Cl}$ & $\mathrm{SO}_{4}$ & $\mathrm{CO}_{3}$ & $\mathrm{HCO}_{3}$ & $\mathrm{SI}$ \\
\hline 1 & 0,007 & 0,003 & 0,004 & 0,003 & 0,003 & 0,003 & 7,1 & 6,1 & 27,4 & 13,1 & 10,7 & 705.1 & 769.2 \\
2 & 0,014 & 0,006 & 0,008 & 0,006 & 0,007 & 0,007 & 6,4 & 6,7 & 25,6 & 14,2 & 5,3 & 836.9 & 895.3 \\
3 & 0,01 & 0,004 & 0,006 & 0,004 & 0,005 & 0,005 & 6,5 & 13,6 & 24,6 & 14,0 & 9,1 & 656.4 & 724.1 \\
4 & 0,016 & 0,007 & 0,009 & 0,007 & 0,008 & 0,008 & 7,6 & 10,3 & 66,3 & 53,1 & 5,3 & 756.4 & 899.1 \\
5 & 0,009 & 0,004 & 0,005 & 0,004 & 0,005 & 0,005 & 6,5 & 4,0 & 38,8 & 30,3 & 10,1 & 646.6 & 736.5 \\
6 & 0,009 & 0,004 & 0,005 & 0,004 & 0,004 & 0,005 & 6,9 & 0,3 & 38,9 & 46,6 & 11,3 & 646.6 & 750.5 \\
7 & 0,009 & 0,004 & 0,005 & 0,004 & 0,004 & 0,004 & 7,3 & 1,5 & 42,1 & 32,8 & 10,7 & 679.5 & 772.7 \\
8 & 0,01 & 0,004 & 0,006 & 0,005 & 0,005 & 0,005 & 8,3 & 2,7 & 30,6 & 25,6 & 8,8 & 560.6 & 636.3 \\
9 & 0,015 & 0,007 & 0,009 & 0,007 & 0,007 & 0,008 & 7,6 & 2,7 & 127,3 & 32,4 & 30,3 & 796.0 & 995.2 \\
10 & 0,015 & 0,007 & 0,009 & 0,007 & 0,008 & 0,008 & 6,8 & 3,6 & 47,5 & 17,5 & 12,9 & 636.2 & 724.4 \\
11 & 0,007 & 0,003 & 0,004 & 0,003 & 0,003 & 0,003 & 6,8 & 1,2 & 15,2 & 14,3 & 0 & 231.8 & 268.9 \\
12 & 0,004 & 0,002 & 0,002 & 0,002 & 0,002 & 0,002 & 6,4 & 0,9 & 18,9 & 14,8 & 0 & 275.7 & 315.9 \\
13 & 0,004 & 0,002 & 0,002 & 0,002 & 0,002 & 0,002 & 6,71 & 0,2 & 18,3 & 13,7 & 1,1 & 395.3 & 434.6 \\
14 & 0,011 & 0,005 & 0,006 & 0,005 & 0,005 & 0,005 & 13,9 & 2,4 & 63,9 & 11,8 & 1,1 & 1122.4 & 1214.9 \\
15 & 0,008 & 0,004 & 0,005 & 0,004 & 0,004 & 0,004 & 9,9 & 1,9 & 23,7 & 17,5 & 0 & 492.9 & 544.9 \\
16 & 0,008 & 0,004 & 0,005 & 0,004 & 0,004 & 0,004 & 12,1 & 0,5 & 24,6 & 14,5 & 0 & 641.7 & 692.9 \\
17 & 0,01 & 0,005 & 0,006 & 0,005 & 0,005 & 0,005 & 14,8 & 2,4 & 39,3 & 15,7 & 0 & 409.9 & 481.4 \\
\hline
\end{tabular}




\section{Análisis de correlación}

El análisis de correlación lineal de Pearson en los sólidos inorgánicos, se ha realizado con el objetivo de establecer el grado de asociación entre las variables analizadas. Para este estudio se ha considerado el número total de muestras estudiadas en las cuarto presas, una laguna y cinco ríos (resultados de los tres muestreos), los nueve metales pesados ( $\mathrm{Co}, \mathrm{Ni}, \mathrm{Fe}, \mathrm{Cu}, \mathrm{Zn}, \mathrm{Mn}, \mathrm{Pb}, \mathrm{Cd}, \mathrm{Cl}$ ) y los tres componentes mayoritarios de los sólidos totales inorgánicos $\left(\mathrm{N}, \mathrm{P}, \mathrm{SO}_{4}, \mathrm{CO}_{3}, \mathrm{HCO}_{3}\right)$.

Los resultados obtenidos tomando como base el análisis factorial (Tabla 3), formaron cinco grupos con diferentes calidades de agua: Grupo I, corresponde a la zona de San Juan Amajac, donde seis componentes principales (nitrógeno, fósforo, sulfatos, carbonatos, bicarbonatos y cloruros) representan el $93 \%$ de variabilidad, de la calidad del agua, la cual esta predominada por sales provenientes de las grutas de Tolantongo. Grupo II, considera la zona de la Cd. De Tulancingo, incluyendo a la presa la Esperanza, fueron siete los componentes principales $\left(\mathrm{N}, \mathrm{P}, \mathrm{SO}_{4}, \mathrm{CO}_{3}\right.$, $\mathrm{HCO}_{3}, \mathrm{Cl}, \mathrm{Mn}$ ), los que explican el $98 \%$ de la variabilidad, de la calidad del agua contaminada proveniente de los vertidos residuales urbanos. Grupo III, considerado como testigo, se establecen cinco componentes principales $\left(\mathrm{N}, \mathrm{P}, \mathrm{SO}_{4}, \mathrm{CO}_{3}, \mathrm{HCO}_{3}\right)$ los cuales representan el $94 \%$ de la variabilidad del agua, clasificada como la mas pura por representar a Presas que se encuentran en la parte alta de la cuenca y las bajas concentraciones de sólidos inorgánicos $(268.9,315.8$ y $434.6 \mathrm{mg}$ $\mathrm{L}^{-1}$, para las presas El Cedral, La Estanzuela y El Comalillo, respectivamente) son de procedencia natural. Grupo IV, corresponde a el agua clasificada a la salida de la Cuenca, la cual incluye el agua del Río Moctezuma, que acarrea aguas negras desde la Ciudad de México, y se representa por cinco componentes principales $\left(\mathrm{N}, \mathrm{P}, \mathrm{SO}_{4}, \mathrm{CO}_{3}\right.$ y $\mathrm{HCO}_{3}$ ) los cuales representan el $96 \%$ de la variabilidad del agua. Grupo V. Corresponde a el agua del Río Venados, Laguna de Meztitlán, y Río Almolón, donde son cinco los componentes $\left(\mathrm{N}, \mathrm{P}, \mathrm{SO}_{4}, \mathrm{CO}_{3}\right.$ y $\left.\mathrm{HCO}_{3}\right)$, que representan el $93 \%$ de la variabilidad en la calidad del agua, que proviene desde El Estado de Puebla, pasando por la Cd. De Tulancingo, Estado de Hidalgo México.

\section{Calidad del agua en el tiempo}

El análisis de la calidad del agua respecto a la variación en el tiempo, indica que la concentración de contaminantes se ve diluida fuertemente con eventos específicos como el paso del Huracán Vilma, en octubre del 2005. En el área se reportaron caudales de hasta $500 \mathrm{~m}^{3}$ por seg-1, arrasando con todo lo que se encontraba a su paso; durante el segundo muestreo (noviembre del 2005), se pudo observar que el agua saco desde la raíz, árboles de hasta $35 \mathrm{~m}$ de altura que se encontraban distribuidos dentro del río. Esto fenómeno se ve reflejado en los resultados obtenidos (Tabla 3), resaltando que los componentes principales son los mismos $\mathrm{N}, \mathrm{P}$. SO4, CO3, y $\mathrm{HCO}$, pero el coeficiente de correlación indica que la mayor asociación se estableció con los elementos pesados $\mathrm{Pb}, \mathrm{Cd}$, Cu y Ni, además del Fósforo.

Tabla 3: Grado de asociación ( $r$ ) entre los componentes principales y los elementos de los sólidos inorgánicos en el agua superficial de la cuenca del Amajac $\left(n=14{ }^{*} p<0,05 ;{ }^{* *} p<0,01\right)$

\begin{tabular}{cccccccc}
\hline Grupo & $\mathrm{N}$ & $\mathrm{P}$ & $\mathrm{SO}_{4}$ & $\mathrm{CO}_{3}$ & $\mathrm{HCO}_{3}$ & $\mathrm{Cl}$ & $\mathrm{Mn}$ \\
\hline $\mathrm{I}$ & $\mathrm{Cu}(0,42)$ & $\mathrm{Ni}(0,51)$ & $\mathrm{Pb}(0,49)$ & $\mathrm{N}(0,63)$ & $\mathrm{P}(0,70)$ & $\mathrm{Mn}(0,63)$ & \\
II & $\mathrm{Cu}(0,43)$ & $\mathrm{Cl}(0,48)$ & $\mathrm{Zn}(0,43)$ & $\mathrm{Cd}(0,40)$ & $\mathrm{SO}_{4}(0,47)$ & $\mathrm{CO}_{3}(0,48)$ & $\mathrm{P}(0,5)$ \\
III & $\mathrm{Pb}(0,42)$ & $\mathrm{Cu}(0,49)$ & $\mathrm{Fe}(0,48)$ & $\mathrm{Zn}(0,62)$ & $\mathrm{P}(0,54)$ & & \\
IV & $\mathrm{N}(0,39)$ & $\mathrm{CO}_{3}(0,45)$ & $\mathrm{Cd}(0,43)$ & $\mathrm{SO}_{4}(0,64)$ & $\mathrm{Mn}(0,42)$ & & \\
V & $\mathrm{Zn}(0,33)$ & $\mathrm{P}(0,45)$ & $\mathrm{Mn}(0,53)$ & $\mathrm{Zn}(0,53)$ & $\mathrm{Pb}(0,84)$ & & \\
Muestra & & & & & & & \\
1 & $\mathrm{Cl}(0,44)$ & $\mathrm{Fe}(0,50)$ & $\mathrm{Cu}(0,49)$ & $\mathrm{Zn}(0,46)$ & $\mathrm{N}(0,35)$ & $\mathrm{Pb}(0,51)$ & \\
2 & $\mathrm{~Pb}(0,42)$ & $\mathrm{Cd}(0,47)$ & $\mathrm{Cu}(0,42)$ & $\mathrm{P}(0,57)$ & $\mathrm{Ni}(0,58)$ & & \\
3 & $\mathrm{Cl}(0,46)$ & $\mathrm{HCO}_{3}(0,5)$ & $\mathrm{N}(0,38)$ & $\mathrm{Cu}(0,5)$ & $\mathrm{Cd}(0,55)$ & $\mathrm{SO}_{4}(0,39)$ & $\mathrm{Cd}(0,5)$ \\
\hline
\end{tabular}

Naddeo et al. (2007), desarrollaron un estudio utilizando la técnica de componentes principales en Italia, para caracterizar la calidad del agua superficial, y pronosticar las posibles consecuencias de un nuevo sitio civil o industrial; Ellos encontraron que la calidad del agua debe incluir los parámetros 
físicos, químicos y biológicos. Hakanson et al. (2005), utilizaron un modelo empírico para predecir la concentración de radionuclidos, nutrientes y metales en agua y biota de los ríos en Suiza, Francia, Hungría, Polonia, Rusia, Suecia y en el Reino Unido, con resultados similares registrados en este trabajo. Ghoualem y Khouider (2008), desarrollaron un estudio, en la zona de Serradla el oeste de Argel, donde el agua se vierte directamente al mar, sin ningún tratamiento previo, registrando que los resultados de la $\mathrm{DBO}_{5}$, turbiedad y conductividad excede fuertemente las normas.

\section{Oxígeno Disuelto}

La mayor contaminación del agua se registro en el río Tulancingo a la altura de la Ciudad de Tulancingo, donde los valores reportados fueron de $0 \mathrm{mg} \mathrm{L}^{-1}$. Sobre el oxígeno disuelto Chapra y Pelletier (2003), establecieron los rangos entre 7 y $8 \mathrm{mg} \mathrm{L}^{-1}$, de oxígeno disuelto como valores que indican aguas libres de contaminación. En el recorrido del río el agua va tomando oxigeno, como se pudo comprobar en la estación de aforo del Río Venados, ubicada a $84 \mathrm{~km}$ de la ciudad de Tulancingo. El agua de la Laguna de Meztitlán Por estar almacenada y con poca aireación, mostro valores de 5,78 $\mathrm{mg} \mathrm{L}^{-1}$, lo cual también esta por debajo de los niveles considerados como adecuados Posteriormente al avanzar el agua recorriendo el cauce del Almolón, y juntarse con las aguas del río Amajac, después del pueblo de Santa María Amajac, los valores del parámetro tratado vuelven a estar dentro de los rangos de agua con buena cantidad de oxígeno disuelto, especialmente por la velocidad que toma el agua (cerca de $1,0 \mathrm{~m} \mathrm{seg}^{-1}$ ), debido a las condiciones físicas del terreno, donde se alcanzan pendientes hasta del 30\%. En un trabajo similar reportado por Parparov (2006), indican valores de 95, 110 y 113 \% de saturación de Oxígeno, en Los Lagos Naroch, Miastro y Batorino, respectivamente.

El análisis de correlación lineal de Pearson, indica que los elementos que se correlacionan linealmente en el agua del río Tulancingo (Tabla 4) son la Temperatura con la turbidez, y el fósforo, con un nivel de significancia de $p<0,01$, como así lo muestra la fuerte dependencia lineal positiva con coeficientes de correlación $r>0,5$. Por otro lado, correlaciones menos acentuadas se dan para la temperatura con la turbidez $(r=0.6989 *, p<0,05)$. Kumar et al. (2008) desarrollaron un estudio para determinar el impacto de la calidad del agua de riego en la calidad del suelo. El análisis de componentes principales identifico la conductividad eléctrica, la conductividad hidráulica y el fósforo disponible como los indicadores más importantes para evaluar la calidad del suelo. Según Marín (2003), el oxígeno disuelto es un gas muy relevante en dinámica del agua, su solubilidad, es función de varios factores: temperatura, presión, coeficiente de solubilidad, tensión de vapor, salinidad y composición fisicoquímica del agua, también reportó que el porcentaje de saturación del oxígeno de una agua, depende de la turbulencia, de la superficie de contacto entre el gas y el agua.

Tabla 4: Matriz de correlación entre los parámetros analizados en el agua del Río Tulancingo $\left(n=14{ }^{*} p<0,05 ;{ }^{*} p<0,01\right)$

\begin{tabular}{|c|c|c|c|c|c|c|c|c|c|}
\hline & OD & $T$ & $\mathrm{pH}$ & Coliformes & DBO & $\mathrm{NO}_{3}$ & $\mathrm{Pt}$ & Turbidez & STD \\
\hline OD & 1 & & & & & & & & \\
\hline $\mathrm{T}$ & $-0,126$ & 1 & & & & & & & \\
\hline $\mathrm{pH}$ & 0,225 & $-0,451$ & 1 & & & & & & \\
\hline Colifo & 0,062 & 0,052 & $-0,141$ & 1 & & & & & \\
\hline DBO & $-0,042$ & $-0,179$ & $-0,014$ & $-0,605^{*}$ & 1 & & & & \\
\hline NO3 & 0,537 & 0,007 & $-0,171$ & $-0,022$ & $-0,192$ & 1 & & & \\
\hline $\mathrm{P}$ & $-0,012$ & $0,747^{* *}$ & $-0,479$ & 0,185 & $-0,498$ & 0,282 & 1 & & \\
\hline Turbidez & $-0,532$ & $0,699 *$ & $-0,566$ & $-0,0209$ & $-0,027$ & $-0,508$ & 0,472 & 1 & \\
\hline STD & 0,077 & 0,106 & $-0,236$ & 0,236 & 0,028 & $-0,373$ & $-0,045$ & 0,323 & 1 \\
\hline
\end{tabular}

\section{Demanda Bioquímica de Oxígeno}

La DBO indica la cantidad de oxígeno necesaria para destruir, estabilizar o degradar la materia orgánica presente en una muestra de agua por la acción bacteriológica. Los resultados obtenidos para la cuenca Amajac . Los valores de demanda bioquímica de oxígeno (DBO 5 ) variaron entre 11,3 y $20,3 \mathrm{mg} \mathrm{L}^{-1}$, para la presa el Comalillo y en el Río Moctezuma, el cual se caracteriza por acarrear las aguas negras provenientes desde la Ciudad de México (Fig. 3). Todos los valores promedio 
fueron inferiores a los LMP de la norma: $150 \mathrm{mg} \mathrm{L}^{-1}$ para uso en riego agrícola, $75 \mathrm{mg} \mathrm{L}^{-1}$ para uso público urbano y $30 \mathrm{mg} \mathrm{L}^{-1}$ para la protección de la vida acuática. Los promedio de la presa "La Esperanza", (11,67 $\left.\mathrm{mg} \mathrm{L}^{-1}\right)$ fueron los más altos, esto debido a la presencia de lirio acuático, en casi el $100 \%$ del espejo del agua. Chapra y Pelletier (2003), establecieron los rangos entre los 12 y $20 \mathrm{mg}$ $\mathrm{O}_{2} \mathrm{~L}^{-1}$, para mostrar que el agua esta contaminada. Bellos y Sawidis (2005), establecieron también que altos valores de DBO, indica contaminación de agua, y cargas significativas de materia orgánica, e incremento de nutrientes y de carbono orgánico, además que la importancia de los procesos depende de factores severos en el ambiente como la temperatura y el pH. Soon y Seok (2002), reportaron que la DBO, es un indicador de la materia orgánica, la cual incluye sólidos que provienen del reino animal o vegetal, o de actividades humanas relacionadas con la síntesis de compuestos orgánicos, pueden ser: proteínas, hidratos de carbono, lípidos, agentes tenso activos, pesticidas y productos químicos de uso agrícola.

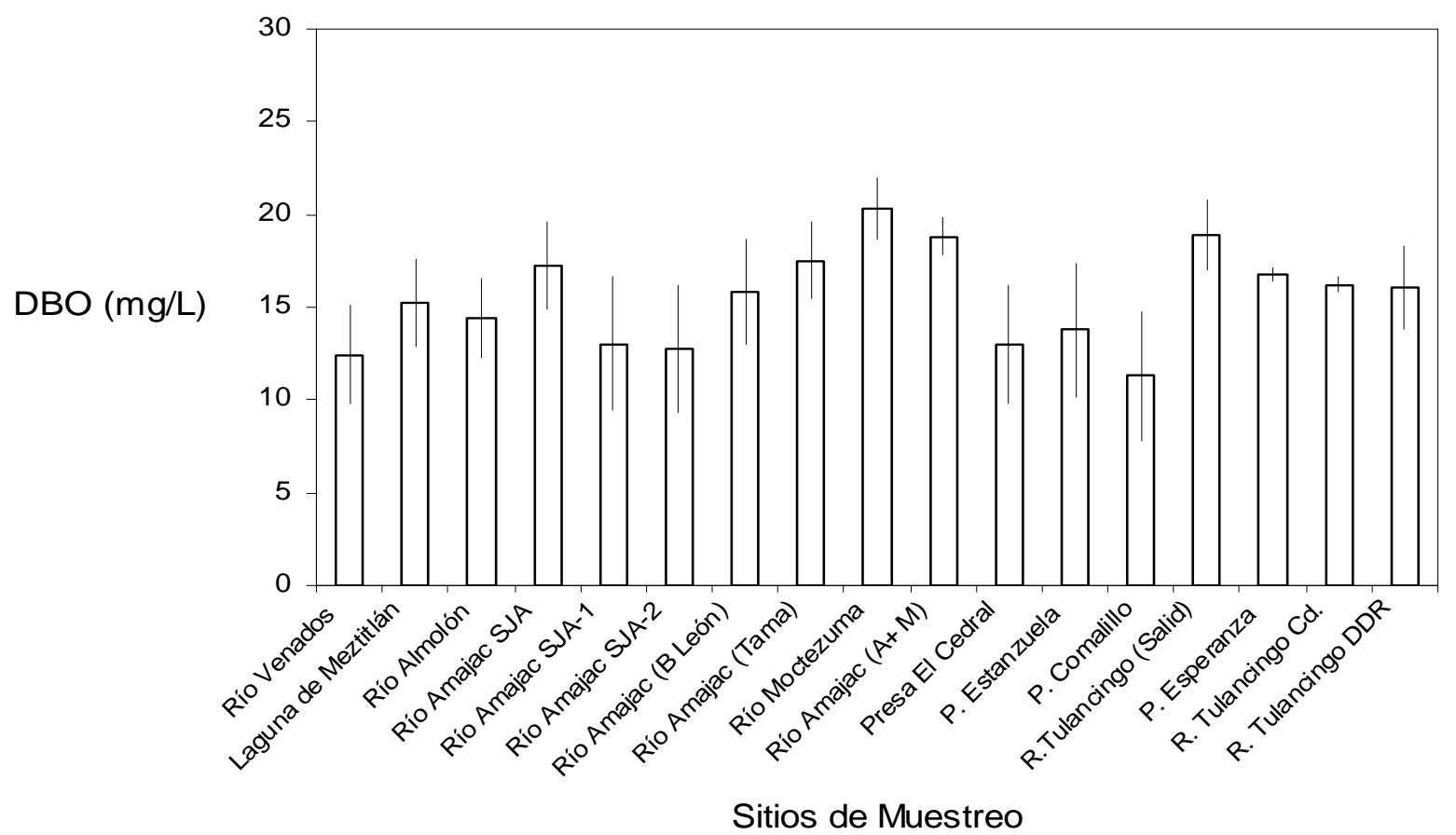

Fig 3: Valores promedio de la $\mathrm{DBO}_{5}$ en aguas superficiales de la cuenca del Amajac. Límite máximo permitido para protección de vida acuática $30 \mathrm{mg} \mathrm{L}^{-1}$.

\section{Coliformes Fecales}

La concentración de coliformes fecales (NMP) osciló entre 106,7 $100 \mathrm{~mL}^{-1}$, para la Presa la Estanzuela, uno de los dos lugares, con valores inferiores a la norma, hasta 24,000 por $100 \mathrm{~mL}^{-1}$, en el Río Tulancingo, donde el agua se encontró altamente contaminada, lo cual supera hasta 12 veces en orden de magnitud los LMP establecidos por la norma (1000 a $2000 \mathrm{NMP} 100 \mathrm{~mL}^{-1}$ ) (Fig. 4). El número más alto de coniformes fecales, debido a las aguas residuales $\left(24,000 \mathrm{NMP} / 100 \mathrm{~mL}^{-1}\right) \mathrm{un}$ valor fuera de la norma. Sin embargo, debe tomarse en cuenta que los valores establecidos por la norma se refieren a aguas tratadas, por lo que no debe sorprender que las concentraciones en las aguas residuales del río Tulancingo, que no reciben ningún tratamiento, sean tan elevadas. Esta variable resultó la más desfavorable al evaluar el grado de contaminación de las aguas del río Tulancingo. Esto implica un riesgo de infección por estos organismos para la población expuesta a los sedimentos secos y suspendidos en el aire. Sobre el tema, De acuerdo con Campos et al. (2000), los gérmenes patógenos como los coliformes fecales, habitualmente transmitidos por el agua, viven en los intestinos del hombre y de los animales de sangre caliente (estos son considerados como agentes de la fiebre tifoidea, del cólera, etc.), e influyen directamente en la contaminación del agua. De acuerdo con Marín (2003), los coniformes fecales están profusamente distribuidos en la naturaleza, por lo que no debe llamar la atención el que todas las aguas naturales contengan una variedad relativamente grande. Algunas de ellas son saprofitas procedentes del suelo, otras pueden ser parásitas. Aun las bacterias patógenas pueden encontrarse en el agua debido a la contaminación 
por detritus de origen humano o animal. Con esta referencia se puede explicar ampliamente el porque, se cuantificaron altas concentraciones en toda la cuenca del Amajac, principalmente en el área de la ciudad de Tulancingo, en el estado de Hidalgo, México.

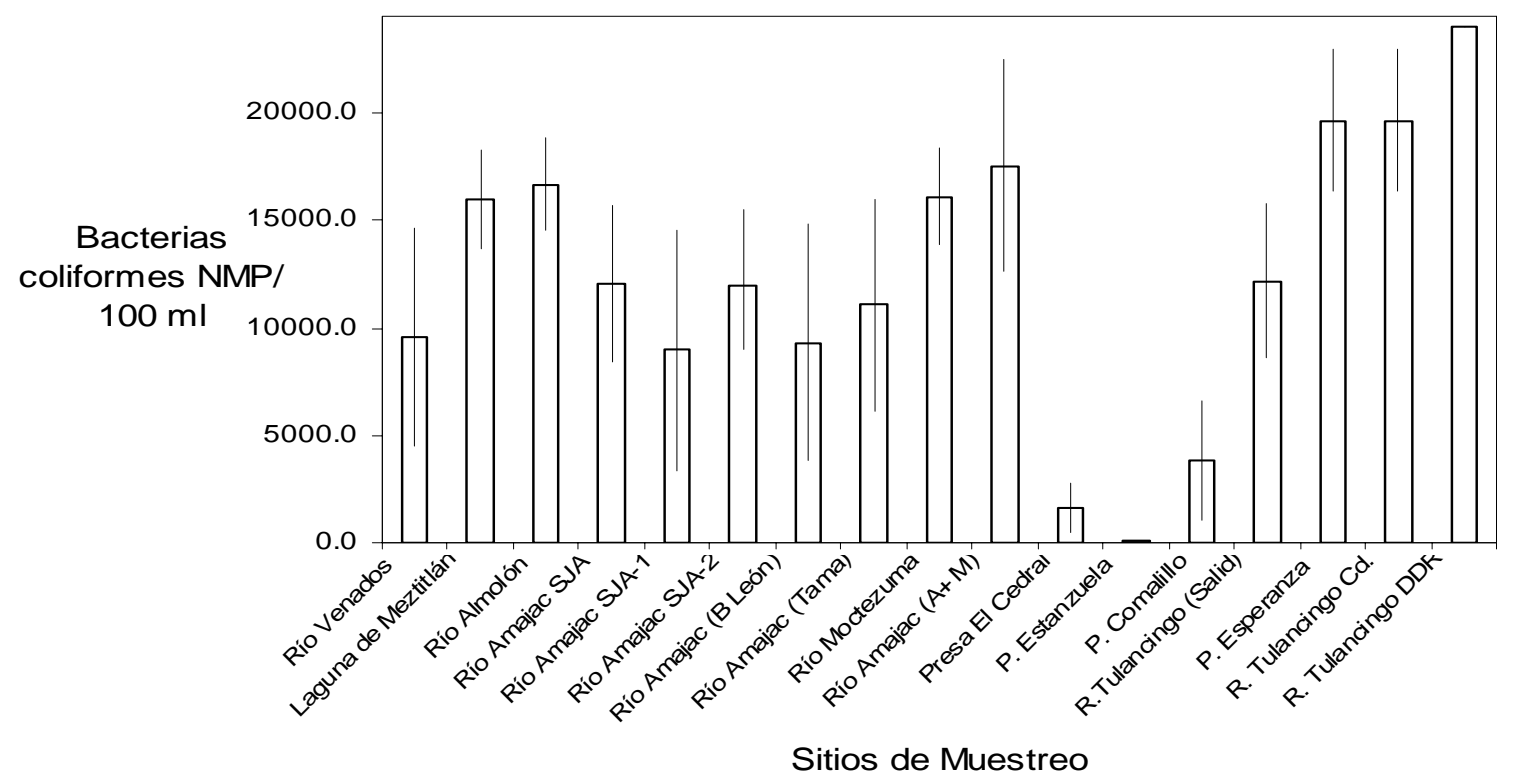

Fig. 4:. Número más probable de bacterias coliformes fecales en aguas superficiales de la cuenca del Amajac. Limite máximo permisible establecidos por la norma 1000 a $2000 \mathrm{NMP} 100 \mathrm{~mL}^{-1}$.

\section{CONCLUSIONES}

La calidad del agua en el río Tulancingo es muy mala, registrando concentraciones de $0 \mathrm{mg} \mathrm{L}^{-1}$ de oxígeno disuelto, mientras que la norma establece como mínimo $10 \mathrm{mg} \mathrm{L}^{-1}$; valores de 24,000 NMP $100 \mathrm{~mL}^{-1}$ de bacterias coliformes fecales lo cual supera 12 veces los valores establecidos en la norma (1000-2000 NMP $100 \mathrm{~mL}^{-1}$ ) y hasta $1,215 \mathrm{mg} \mathrm{L}^{-1}$ de sólidos inorgánicos, siendo que la norma oficial establece los límites de $100 \mathrm{mg} \mathrm{L}^{-1}$, lo cual indica fuerte grado de contaminación. La presa La Esperanza de Tulancingo, Hidalgo se encuentra en estado avanzado de eutrofización, ya que el lirio acuático que se presenta el $100 \%$ del espejo del agua de esta presa. Las aguas del Río Amajac están libres de contaminantes pueden utilizarse para todos los usos como las actividades agrícolas, engendrar peces y producción en hidroponía bajo condiciones de invernadero y equivalen a una tercera parte de las concentraciones registradas en el río Tulancingo. El análisis aplicado al estudio indica que los componentes principales que representan la calidad integral del agua dentro de la cuenca estudiada son: oxígeno disuelto, coliformes fecales, nitrógeno, fósforo, sulfatos, carbonatos, bicarbonatos, cloro y manganeso, los cuales explican más del $90 \%$ de la variabilidad total.

\section{AGRADECIMIENTOS}

Al CONACYT, y al Gobierno del Estado de Hidalgo, los cuales a través del financiamiento del proyecto Fondos Mixtos CONACYT- HIDALGO 8848 Titulado "Ordenación de Recursos Hídricos del Río Amajac (subcuenca del pánuco) relación socioeconómica y ambientales en el Estado de Hidalgo, hizo posible el desarrollo del mismo.

\section{REFERENCIAS}

Acosta, E. H., y otros cuatro autores; "Contaminación del agua en el río Tulancingo, Estado de Hidalgo, México".In: MEDIOAMBIENTE EN IBEROAMERICA. Visión desde la Física y la Química en los albores del Siglo XXI. Tomo I, por F Gallardo, pp: 335-341. Sociedad Iberoamericana de Física y Química Ambiental. Badajoz, España (2006) 
Ayala, C. R., y otros cuatro autores; "Cantidad y calidad química del agua en el río Amajac".In: MEDIOAMBIENTE EN IBEROAMERICA. Visión desde la Física y la Química en los albores del Siglo XXI. Tomo I.,por F Gallardo, pp: 379 385.Sociedad Iberoamericana de Física y Química Ambiental. Badajoz, España.(2006)

Briones, S. G. e I. García, C; Aforo del agua en canales y tuberías. $1^{\text {a }}$ ed. Editorial Trillas, México, D. F.(1997)

Bellos, D. y T. Sawidis; Chemical pollution monitoring of the River Pinios (Tesalia-Greece). Journal of Environmental Management: 76, 282-292 (2005)

Campos, C., G. Orno, M. Salgot e Y L. Gillerman; Behaviour of the fecal pollution indicators in soil irrigated with treated wastewater under on surface and subsurface drip irrigation. Water Science and Technology: 42(1-2), 75-79. (2000)

Chapra, S.C. y G.J. Pelletier. QUAL2K: A Modeling Framework for Simulating River and Stream Water Quality: Documentation and User Manual. Civil and Environmental Engineering Dept., Tufts University, Medford, M. A., Steven. Chapra @ tufts, edu. (2003)

Frutos, S.M.; Densidad y diversidad del zooplancton en los ríos salado y negro- planicie del río Paraná-Argentina. Centro de Ecología Aplicada del Litoral (CECOAL-CONICET), Corrientes, Argentina (1998)

Foreman, M.G.G., y otros cuatro autores; Flow and temperature models for the Fraser and Thompson rivers. Atmos. Ocean.: 35,109-134. (1997)

Ghoualem, H. y A. Khouider; Evaluation and biologic treatment of an urban effluent. Desalination: 222(1-3), 302-306 (2008)

Hakanson, L., y otros cuatro autores; Development of a system of water quality as a tool for management. Final report to INTAS, Uppsala University, Department of Earth Science: 19,7-11. (2000)

Hakanson L., M. Mikrenska, K. Petrov, y I. Foster; Suspended particulate matter (SPM) in rivers: empirical data and models. Ecological Modelling; 183,251-267 (2005)

INEGI. Pachuca. Carta Topográfica. F14D81-(1999), Tulancingo Carta topográfica F14D82- (1999) Actopan, Carta topográfica. F14D71-(2000) Metztitlan. Carta Topográfica. F14D61-(2000). Molango. Carta Topográfica. F14D51-(2000). Carbonero Jacales. Carta Topográfica. F14D72-(2001), San Nicolás. Carta Topográfica. F14C59-(2001), Zacualtipán. Carta Topográfica. F14D62-(2001), Jacala. Carta topográfica. F14C49-(2002), Chapuluacan. Carta Topográfica. F14D41- (2003). Aguascalientes, Ags. México.(2003)

INEGI; XIII. Censo General de Población y Vivienda. Aguascalientes, Ags. México. (2005)

Jones, P.D., A.O. Tyler y A.W. Wither; Decision Support Systems: Do they have a future in Estuarine Management? Estuarine Coastal Shelf Sci.: 55,993-1008.(2002)

Jones, P.D.; Water quality and fisheries in the Mersey estuary, England: A historical perspective. Marine Pollution Bulletin: 53, 144-154.(2006)

Justic, D., N.N. Rabalais y R.E. Turner; Effects of climate change on hypoxia in coastal waters: A doubled $\mathrm{CO}_{2}$ scenario for the northern Gulf of Mexico. Limnol. Oceanogr.: 41, 992-1003. (1996)

Kretschmr, N., L. Ribbe y H. Gaese; Wastewater Reuse for Agriculture. Tecnology Ressource Management and Development- Scientific Contribution for Sustainable Development: 2, 37-64. (2002) 
Kumar, M.U. y otros seis autores; Evaluating impacts of irrigation water quality on a calcareous clay soil using principal component análisis. Geoderma: 144(1-2), 189-197 (2008)

László, B. y otros cuatro autores; Implementation of the EU Water Framework Directive in monitoring of smallwater bodies in Hungary, I. Establishment of surveillance monitoring system for physical and chemical characteristics for small mountain watercourses. Microchemical Journal: 85(1), 65-71 (2007)

Marín, G.R.; Fisicoquímica y microbiología de los medios acuáticos tratamiento y control de calidad de aguas. $1^{a}$ ed. Editorial Díaz de Santos. Universidad Politécnica de Cartagena. Cartagena, España. (2003)

Naddeo V., T. Zarra y V. Belgiorno; Optimization of sampling frequency for river water quality assessment acording to Italian implementation of the EU Water Framework Directive. Environmental Science \& Policy: 10(3), 243-249 (2007)

Panta, J.E.R., y otros cuatro autores; "Aforo y calidad del agua en el rúo Tulancingo, Estado de Hidalgo, México".In: MEDIOAMBIENTE EN IBEROAMERICA. Visión desde la Física y la Química en los albores del Siglo XXI. Tomo I.,por F Gallardo, pp: 401-407 Sociedad Iberoamericana de Física y Química Ambiental. Badajoz, España.(2006)

Parparov, A., K.D. Hambright, L. Hakanson y A. Ostapenia; Water quality quantification: basics and implementation. Hidrobiologia: 560, 227-237 (2006)

Sharma, A. y N. Ashwath; Land disposal of municipal effluents: importance of choosing agroforestry systems. Desalination: 187, 361-374 (2006)

Sawidis, T.; Chemical pollution monitoring of River Pinios in the Mediterranean climate region. Toxic I. Environmental Chem.: 62, 217-227. (1997)

Secretaría de Economía; Establece los límites máximos permisibles de contaminantes en las descargas de agua residuales en aguas y bienes nacionales. NOM-001-SEMARNAT-1996. Diario Oficial de la federación. 6 de enero de 1997. México. 15 p.(1996)

Taylor, K.S. y otros cuatro autores; Subsurface driplinetubing-an experimental design for assessing the effectiveness of using dripline to apply treated wastewatere for turf irrigation in Western Australia. Desalination: 187, 375-385 (2006)

SAS Institute Inc. Release 8.02. SAS Institute Inc. Cary NC 27513, USA (2001)

Soon, P.S. y Seok, Y.L.; A water quality modeling study of the Nakdong River, Korea. Ecological Modelling: 152, 65-75. (2002) 